\title{
Essay
}

\section{Parasitology as a Teaching Tool: Isolation of Apicomplexan Cysts from Store-bought Meat}

\author{
Tracy L. Eggleston, ${ }^{* \dagger}$ Eileen Fitzpatrick, ${ }^{\S}$ and Kristin M. Hager ${ }^{* \dagger}$
}

\author{
*Department of Biological Sciences, University of Notre Dame, Notre Dame, IN 46556-0369; ${ }^{\dagger}$ Center for \\ Global Health and Infectious Disease, University of Notre Dame, Notre Dame, IN 46556-0369; and \\ §Washington High School, South Bend, IN 46601
}

Submitted August 23, 2007; Revised March 3, 2008; Accepted March 12, 2008

Monitoring Editor: Barbara Schulz

\section{INTRODUCTION}

There are obstacles to teaching science; however, these obstacles are not insurmountable. One obstacle is the students themselves. Students often labor under the misconception or anxiety that the course material will be too difficult to understand, or boring (mind-numbing), or that the information learned will not be applicable in their day-to-day lives. Herein lies the challenge for the educator; how can laboratory experiments be designed that are educational, interesting, and accessible for introductory biology or nonmajors students? Our short-term goals in designing this type of experiment were as follows: 1) create a laboratory module accessible to college and high school advanced placement (AP) students that 2) uses parasitology, 3) follows Occupational Safety and Health Administration biosafety guidelines, and 4) meets national secondary school educational guidelines. Specifically, our module is designed to meet the national standards set by the National Academies of Science National Committee on Science Education Standards and Assessment for secondary schools for the following topic areas: science as inquiry, interdependence of organisms, personal and community health, and science as a human endeavor (National Committee on Science Education Standards and Assessment, 1996).

\section{PARASITOLOGY AS A TEACHING TOOL}

Parasitology - the intimate association of two species where one species (the parasite) benefits at the expense of the other (the host) - is referenced throughout the history of humankind. Initially, the study of parasites was restricted to a discussion of symptoms caused by a particular disease. For example, ancient Egyptian hieroglyphs exist that represent

DOI: $10.1187 /$ cbe.07-08-0062

Present address: ${ }^{\ddagger}$ Department of Anatomy and Physiology, Indiana University School of Medicine, Indianapolis, IN 46202.

Address correspondence to: Kristin M. Hager (khager1@nd.edu). infection with Schistosoma hematobium (Marquardt et al. 2000). The glyph depicts infection by showing male genitalia passing a small stream of urine. It translates as a cry of pain and describes the symptom of painful urination experienced by infected individuals (Marquardt et al., 2000). The symptoms of parasitic infection are also written about in historical medical texts. A 1700 BC, Chinese document discusses malaria, and Hippocrates described the three fevers associated with malaria as quotidian, tertian, and quartan. Additionally, clear clinical descriptions of infections caused by worms (helminthes) are given by the physician Paulus Aeginta (AD 625-690; Cox, 2002). Parasitology advanced from a discussion of symptoms to study of the parasites themselves. Protozoa were first visualized in the late 1600s by Antony van Leeuwenhoek (Dobell, 1960). Leeuwenhoek, a Dutch merchant, used a set of specialized lenses of his devising, to visualize the first microscopic organisms called protozoa (Dobell, 1960). Leeuwenhoek's work contributed to the formulation of a very basic and powerful cell biology tenet called the Cell Doctrine. The Cell Doctrine, or Cell Theory, formally articulated by Schleiden and Schwaan in 1839 , states that all organisms are composed of similar units of organization called cells. Since these early studies, large strides have been made in parasitology. Furthermore, parasites have proved to be strong model systems for understanding many basic biological principles. For example, in the nineteenth century, the continuity of chromosomes was demonstrated in parasitic nematodes, and in the twentieth century the structural details of glycosylphosphatidylinositol (GPI) anchors were first elucidated in variant surface glycoprotein coat of kinetoplast parasites (Ferguson et al., 1988). Consequently, parasitology has a long history of association with the study of cell biology and represents a powerful teaching tool for introducing both basic and advanced biological concepts.

Parasites are extremely prevalent not only in terms of historical medical and research references but also in terms of geographic distribution. Human parasites, such as Trichuris trichiura, Ascaris lumbricoides, and hookworms have re- 
mained relatively unchanged in their geographic distribution in the past $50 \mathrm{yr}$ (Roberts and Schmidt, 2000). An outstanding example of this widespread distribution is the protozoan parasite Toxoplasma gondii. T. gondii infects humans worldwide and is broadly distributed in animals as varied as polar bears, bobcats, pigs, and even in marine mammals such as seals and otters (Fayer et al., 2004). In practical terms, this cosmopolitan distribution means that $T$. gondii is readily isolated from raw meat purchased at a local grocery store and thus a parasitology lesson is as close as your nearest checkout line.

\section{ROLE OF PARASITOLOGY IN STUDYING THE HEALTH, SAFETY, AND MICROBIOLOGY OF FOOD}

Parasites are widely dispersed across domesticated livestock or feed animals (Fayer et al., 2004). The eggs of some helminthes and certain protozoan parasites are passed in the feces of these animals. The oocysts of the protozoan parasite T. gondii are shed exclusively by domesticated and feral cats. Wild and domesticated animals contribute trillions of kilograms of feces that can pollute the water supply (Fayer et al., 2004). Two recent studies of water used to irrigate vegetables in the United States and several Central American countries found that water was polluted with livestock feces and tested positive for both Giardia and Cryptosporidium parasites (Thurston-Enriquez et al., 2002; Fayer et al., 2004; ThurstonEnriquez et al., 2005). Consequently, potential sources of parasitic infection from our food supply are not limited to meat. T. gondii infects humans through many different routes, such as ingesting undercooked meat, contaminated food, or contaminated water (Roberts and Schmidt, 2000). Due to its ubiquitous distribution, it is not surprising that $T$. gondii is the third leading cause of food-borne disease (Gardner, 2004).

\section{TOXOPLASMA IS AN APICOMPLEXAN PARASITE COMMONLY FOUND IN STORE-BOUGHT MEAT}

T. gondii is an obligate intracellular parasite capable of invading virtually any nucleated cell. It belongs to the phylum Apicomplexa, which includes Plasmodium falciparum (the causative agent of malaria), Neospora caninum and Sarcocystis cruzi (cattle pathogens), and $\sim 5000$ other named species (Levine, 1988). Two specialized secretory organelles called micronemes and rhoptries are in the anterior cytoplasm of apicomplexan parasites. This complex of specialized substructures gives the phylum its name (Levine, 1977). Several apicomplexans also possess another distinctive organelle called the apicoplast (McFadden et al., 1996; Köhler et al., 1997; Lang-Unnasch et al., 1998; Zhu et al., 2000; Abrahamsen et al., 2004). This organelle is apical, plastid in nature, and thought to have evolved through secondary endosymbiosis (Kohler et al., 1997; Waller and McFadden, 2005). Unlike other apical organelles, it harbors its own genome, and it is enclosed within four membranes (Kohler et al., 1997; McFadden and Roos, 1999). Whereas some related Apicomplexa such as $P$. falciparum possess complex multistage life cycles, $T$. gondii has a relatively simple life cycle that can be broken down into two stages, sexual and asexual (Figure 1). The sexual stage occurs in cats, the definitive host for $T$. gondii (Omata et al., 1997). Cats shed oocysts in their feces whereby they contaminate the food and water of livestock (Roberts and Schmidt, 2000; Figure 1). The livestock become intermediate hosts where oocysts divide. Oocysts divide asexually, and this division occurs primarily in the muscle and brain of
Figure 1. Life cycle of T. gondii. There are three infectious stages of the parasite $T$. gondii: the fast-growing form of the parasite called tachyzoites; the slow-growing form of the parasite called bradyzoites (found in tissue cysts); and the stage released in the feces of the cat, called oocysts. The definitive host (where sexual recombination occurs) is the cat. T. gondii can be transmitted vertically from mother to fetus and cause birth defects and spontaneous abortion. This figure is courtesy of Dubey et al. (1998).

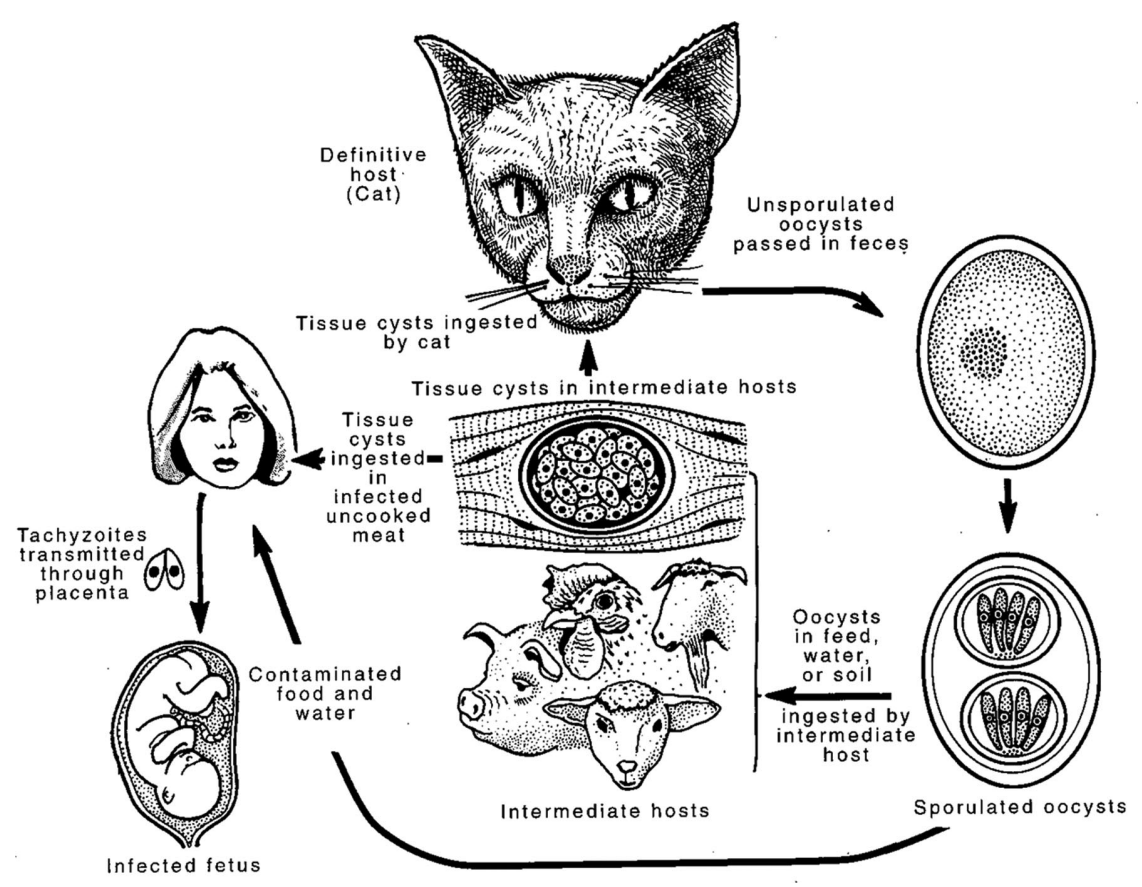


the host (Omata et al., 1997; Figure 1). The livestock do not shed the T. gondii oocysts in their feces. T. gondii cysts are commonly found in livestock such as sheep, chicken, and pigs (Figure 1). Humans are infected through gardening or ingesting contaminated food or water supplies (Gardner, 2004; Figure 1). It is estimated approximately one-third of the world population is currently infected with T. gondii (Hill et al., 2005).

T. gondii is classified as an opportunistic pathogen. This means a healthy immune system normally keeps the parasite in check (Black and Boothroyd, 2000). Occasionally even weak infections can have severe consequences, such as when Martina Navratilova contracted a mild case of toxoplasmosis and lost the 1982 U.S. Open tennis tournament (Roberts and Schmidt, 2000). The parasite usually saves its most devastating effects for the defenseless members of our society. All these members have a common denominator, a poorly functioning immune system. It affects AIDS patients; patients receiving immune-suppressing drugs to prevent rejection of an organ after it is transplanted; and, tragically, our next generation-fetuses and newborns (Black and Boothroyd, 2000; Roberts and Schmidt, 2000). There is no cure, nor is there a human vaccine. Current treatments stop advancement of the disease; however, the side effects are often severe.

The primary focus of this essay is on the development of an assay to isolate apicomplexan cysts. We believe as an outcome of using this assay, teachers will be able to use the isolation of Toxoplasma and related apicomplexan tissue cysts as a means to teach food safety, and to introduce some basic concepts about infectious disease and cell biology. Additionally, this laboratory exercise is an outstanding way to initiate a discussion about the 26-year AIDS epidemic and its profound effects (life ending), especially in developing countries. The National Association of Biology Teachers reiterates the importance of this topic by stating that "biology educators must play a central role in addressing the spread of HIV and the global threat of AIDS."

T. gondii is classified as a biosafety level 2 (BSL-2) pathogen by the Centers for Disease Control and Prevention (CDC). Therefore, isolation of T. gondii is recommended only for those laboratories possessing a biosafety hood and an instructor trained in the handling of BSL-2 hazardous materials. High school laboratories are strongly discouraged from isolating BSL-2 pathogens. For those teachers who want to use this protocol with minors, we suggest isolating a different apicomplexan tissue cyst, such as N. caninum, from cattle. In contrast to the majority of warm-blooded mammals, cattle seem to be resistant to cyst formation by Toxoplasma (Innes et al., 2007). Isolation of N. caninum is recommended for high school laboratories because there is no strong evidence that $N$. caninum can infect or cause disease in people (Graham et al., 1999; Dubey et al., 2007; Innes et al., 2007). In contrast, N. caninum is a major cause of reproductive failure in cattle (Dubey et al., 2007). Because N. caninum is an apicomplexan, this procedure still allows the teacher to introduce topics on related Apicomplexa such as T. gondii.

\section{MATERIALS AND METHODS}

The materials and methods detailed below should allow instructors to conduct this experiment in their own classrooms. We highly suggest practicing this exercise several times before implementation in the classroom. Each aspect can, and should, be evaluated for appropriateness and ease of use within the strictures of each individual biology curriculum (in terms of class size, budget, supplies, and time allotted for the lab). We list the major supplies used in this experiment. A more comprehensive list, including the prices of items, is in an Excel spreadsheet in Supplemental Material Table 1. We include a special subsection for introductory approaches more suitable for high school laboratories. Note that the supplies should be enough to last for years, and several of the supplies could potentially be used in other experiments.

\section{Supplies and Suppliers}

Supplies. One liter of methanol (EF467120RAD; A. Daigger, Vernon Hills, IL), $250 \mathrm{ml}$ of Percoll (17089102; GE Healthcare, Piscataway, $\mathrm{NJ}), 500 \mathrm{~g}$ of potassium chloride (KCl; BP366-500; Fisher Scientific, Pittsburgh, PA), $500 \mathrm{~g}$ of sodium phosphate dibasic heptahydrate $\left(\mathrm{Na}_{2} \mathrm{HPO}_{4} \cdot 7 \mathrm{H}_{2} \mathrm{O}\right.$; BP331-500; Fisher Scientific), $500 \mathrm{~g}$ of potassium phosphate monobasic $\left(\mathrm{KH}_{2} \mathrm{PO}_{4}\right.$; BP362-500; Fisher Scientific), 500 $\mathrm{ml}$ of Tween 80 (T164-500; Fisher Scientific), $5 \mathrm{~g}$ of trypsin (T47995G; Sigma-Aldrich, St. Louis, MO), $50 \mathrm{~g}$ of ethylenediaminetetraacetic acid (EDTA; E5134-500; Sigma-Aldrich), $10 \mathrm{~g}$ of Giemsa stain (11700-10G; Sigma-Aldrich), and 15-ml centrifuge tubes (S50712; Fisher Scientific).

\section{Safety and Biosafety Considerations}

Meat Selection. T. gondii is classified as a BSL-2 hazard. As stated above, isolation of T. gondii cysts or parasites is only suitable for those laboratories that possess a biosafety hood and an instructor trained in the handling of BSL-2 hazardous materials. Teachers who want to use this protocol with minors are urged in the strongest possible terms to avoid BSL-2 pathogens. Therefore, we advise using beef. Do not use lamb or pork for cyst isolation with minors. We recommend isolating $N$. caninum tissue cysts from beef (cattle) for laboratories in secondary schools because there is no strong evidence that $N$. caninum can infect or cause disease in people (Graham et al., 1999; Dubey et al., 2007; Innes et al., 2007). Note that studies have shown that T. gondii can infect cattle (More et al., 2007); however, it does not seem capable of forming cysts in detectable amounts (Innes et al., 2007).

Aerosol Hazards. We suggest using a blender for homogenizing the meat. The blender creates aerosols. To decrease exposure to aerosols, we recommend placing the blender in a hood. To further reduce the aerosol risk, the blender step can be replaced. Instead of using a blender, glass beads (marble sized) can be placed in a clean, plastic screw-capped tube or container along with the meat and buffer. The lid is then replaced, and the container is shaken. This method is almost as effective as the blender.

Contact Hazards. Methanol is a contact hazard. Giemsa can be an allergen. Always practice best lab safety: wear gloves, a lab coat, and goggles. To reduce exposure to methanol, lower slides into a beaker that is prefilled with methanol (Figure 2i) by using tweezers.

\section{Tissue Cyst Isolation: Advanced Approaches (Suitable for College)}

Obtaining, Evaluating, and Placing Meat into Aliquots. Lamb, ground beef, and pork sausage were purchased from a local grocery store. The connective tissue was trimmed off using razor blades. The meat was then cut into pieces $(\sim 1 \times 1 \mathrm{~cm})$ and placed into $5-\mathrm{g}$ aliquots (Figure 2a). We wore gloves, a lab coat, and goggles. All items that touched the raw meat were rinsed with bleach (straight) and disposed of in a garbage bag. The razors were disposed of in a sharps container.

Homogenizing Meat. Each 5-g portion of meat was homogenized by resuspending the meat into $100 \mathrm{ml}$ of phosphate-buffered saline/ $0.1 \%$ Tween 80 buffer, pH 7.4 (PBS/Tween 80) (Omata et al., 1997). The resultant resuspension was then placed in a blender and pulsed for $2 \mathrm{~min}$ (Blewett et al., 1983). The homogenized meat was poured 
into a beaker. The blender was washed with an additional $5 \mathrm{ml}$ of PBS/Tween 80 to remove any remaining debris, and the wash solution was then added to the meat homogenate (Figure $2 b$ ). The homogenate (mechanically disrupted tissue in solution) was then filtered through two layers of gauze or cheesecloth three times in total (Figure 2c; Dubey et al., 1998; Garcia et al., 2006). The filtrate is collected and saved for the next step.

Percoll Gradient. Each homogenate was subjected to a low-speed spin $(\sim 500 \times g)$ for $5 \mathrm{~min}$. After centrifugation, $800 \mu \mathrm{l}$ of each supernatant (Figure 2d) was added to a 1.5-ml Eppendorf tube (Cornelissen et al., 1981; Blewett et al., 1983; Omata et al., 1997). A 2-ml disposable serologic pipette was used to underlay $250 \mu \mathrm{l}$ of $30 \%$ Percoll below the homogenate (Figure 2e, left). A second layer of $250 \mu \mathrm{l}$ of $90 \%$ Percoll was then underlaid below the 30\% layer (Blewett et al., 1983). The resultant system contained, from top to bottom, $800 \mu \mathrm{l}$ of meat supernatant (postcheesecloth filtration $250 \mu \mathrm{l}$ of $30 \%$ Percoll, and $250 \mu \mathrm{l}$ of $90 \%$ Percoll (Figure 2e, drawing). All Percoll dilutions were done in PBS.
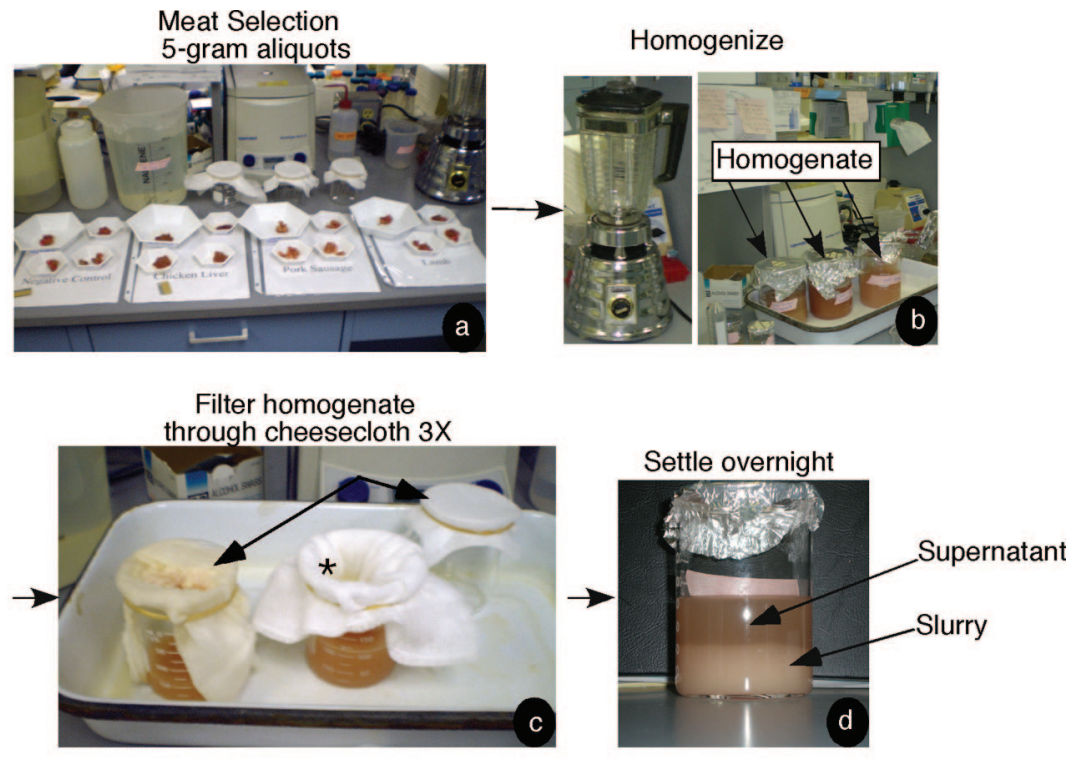

Figure 2. Schematic of strategy used to enrich for apicomplexan cysts. We show here a series of pictures demonstrating the protocol in a stepwise manner. For more experimental details, please refer to Materials and Methods. (a) Meat selection. Meat was chosen, cut into small pieces $(1 \times 1 \mathrm{~cm})$ and placed in aliquots according to meat category. (b) Homogenize. The meat was resuspended and subjected to mechanical breakdown (either by blender or a container with glass beads). (c) Filter homogenate though cheesecloth three times (3X). The meat homogenate was then subjected to three rounds of filtration through cheesecloth. The homogenate was poured through cheesecloth, collected, and filtered an additional two times. (d) Settle overnight. The supernatant is separated from the meat slurry either by centrifugation or by overnight incubation (refrigerator). (e and f) Percoll gradient or sucrose flotation. The supernatant was subjected to either gradient analysis (Percoll) or flotation (sucrose). The cysts are less dense than other large parasite eggs or the parasites themselves. Therefore, the cysts and eggs float to the top of sucrose or migrate to their known density in the Percoll gradient (white arrow). ( $g$ and $h$ ) Slide preparation. Prepare slide by placing a drop of the isolated cysts onto the slide. Use second slide to create sample smear. (i) Slide staining. After the samples dried, they were fixed in methanol (1), stained with Giemsa (2), and rinsed two times (3 and 4 ) in distilled water, and allowed to dry.
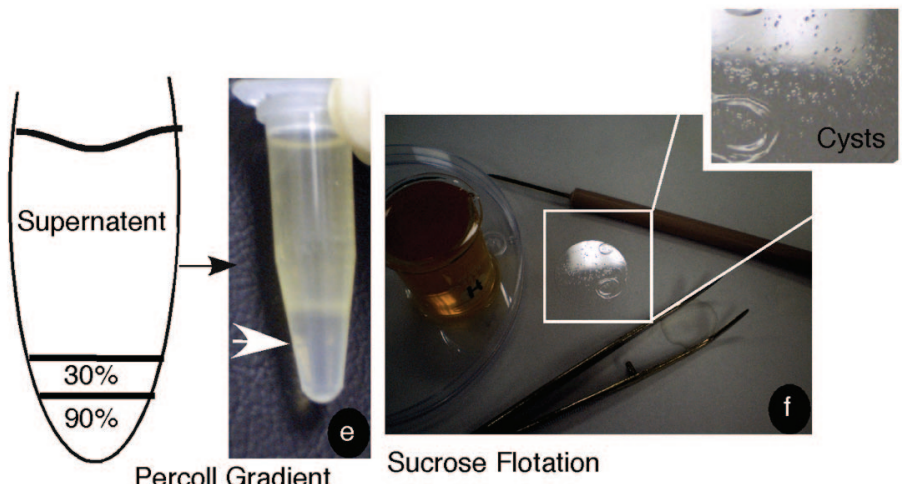

Percoll Gradient

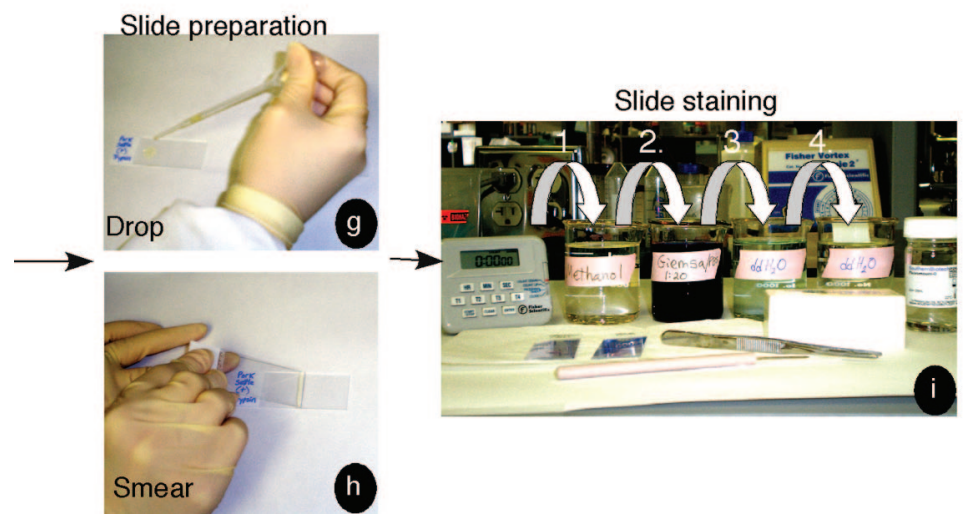


The sample was then centrifuged for $15 \mathrm{~min}$ at room temperature at $\sim 4000 \times g$ in a microcentrifuge (Blewett et al., 1983). The resultant sample is shown in Figure 2e (right). From top to bottom of the Eppendorf tube, we observed the supernatant, a bacterial mat (observed as a light yellow/white band), and the Percoll gradient (colorless). The desired sample (Figure 2e, white arrow) lies underneath the bacterial mat, contains between 60 and $90 \%$ Percoll, and is enriched in T. gondii cysts (Cornelissen et al., 1981; Blewett et al., 1983). The layer containing the $60-90 \%$ Percoll (Figure 2e, white arrow) was collected with a $2-\mathrm{ml}$ serologic pipette. The pipette was slowly inserted into the tube, being careful not to disturb the mat. Once the tip of the pipette reached the bottom of the tube, a gentle suction was applied to collect the bottom part of the gradient. The final sample is $\sim 300 \mu \mathrm{l}$ in volume. A more accurate density determination of Percoll gradients is ascertained using an external standard such as a centrifuge tube containing density marker beads run in parallel to the experimental sample. Should an instructor want to use beads, the beads should be used according to manufacturer's protocol (kit DMB-10-1KT [\$387.50]; Sigma-Aldrich).

\section{Microscopy}

One drop of the suspension was transferred to a clean, dry microscope slide by using a disposable Pasteur pipette (Figure 2g). To release bradyzoites from collected $T$. gondii cysts, a PBS solution containing $25 \%$ trypsin (wt/vol) and $5 \mathrm{mM}$ EDTA was added to the sample drop on the slide. The resultant mixture was incubated at $37^{\circ} \mathrm{C}$ for $5 \mathrm{~min}$. The slide was then air-dried for an additional $30 \mathrm{~min}$ at room temperature (Blewett et al., 1983). The isolated cysts manifest as a fine layer of small grayish particles, sand or grit-like in texture or appearance when dried onto glass slides or coverslips (Figure 2f, inset). The slide was fixed in methanol for $5 \mathrm{~min}$, stained in a 1:20 dilution of Giemsa stain (1 $\mathrm{g}$ of Giemsa in $19 \mathrm{ml}$ of PBS) for 30 min (Blewett et al., 1983; Omata et al., 1997), and then it was rinsed two times in double-distilled water (Figure 2i). The stained sample was air-dried for $15 \mathrm{~min}$. Coverslips were mounted on the slides by using a small drop of mounting media $(\sim 20-30 \mu \mathrm{l})$. Samples were viewed under the microscope (DM IRE 2; Leica, Deerfield, IL) by using a $40 \times$ objective (lower powers can be used). Images were captured by an Orca extended range camera (a blackand-white camera) at $1 \times 1$ binning, 1300 by 1030 pixels, as described previously (Pfluger et al., 2005). We also prepared two additional slides: one slide was a sample without trypsin treatment (to observe unbroken cysts); the other was a slide that had been mock treated with buffer.

\section{Tissue Cyst Isolation: Introductory Approaches (Suitable for AP High School Biology)}

Day 1 ( $\sim 50 \mathrm{~min})$.

Safety Considerations. Be sure to use gloves, a lab coat, and goggles. All items that touch raw meat should be rinsed with bleach (straight) and disposed of in a garbage bag. Methanol is a contact hazard, and it should be disposed of in an approved container. Giemsa is an allergen. Be sure to consult the safety and biosafety considerations section discussed above. In brief, do not use lamb or pork for cyst isolation with minors-choose beef.

Creation of Homogenate (Figure 2, a-c). To creat the homogenate, perform the following steps:

1. Purchase ground beef from a local grocery store.

2. Use scissors to cut meat into small pieces $(\sim 1 \times 1 \mathrm{~cm})$.

3. Label weigh boats (type of meat chosen and name of student group).

4. Weigh meat, and divide it into 5-g aliquots (Figure 1a).

5. Resuspend meat in $100 \mathrm{ml}$ of homogenization buffer (PBS/ Tween 20 or Tween 80 [1.0\%, wt/vol]). Note that the key to the homogenization buffer is that it is a nonionic detergent (Tween part of the buffer). The ratio of meat to buffer is 1:20 wt/vol. Note that $\mathrm{wt} / \mathrm{vol}$ is a measurement ratio. For example 1:20, wt/vol: $1 \mathrm{~g}$ and adjust the volume to $20 \mathrm{ml}$. To make PBS, dissolve the following in $800 \mathrm{ml}$ of distilled $\mathrm{H}_{2} \mathrm{O}: 8 \mathrm{~g}$ of $\mathrm{NaCl}$, $0.2 \mathrm{~g}$ of $\mathrm{KCl}, 1.44 \mathrm{~g}$ of $\mathrm{Na}_{2} \mathrm{HPO}_{4}$, and $0.24 \mathrm{~g}$ of $\mathrm{KH}_{2} \mathrm{PO}_{4}$, mix well, and then adjust $\mathrm{pH}$ to 7.4. Adjust volume to 1 liter with additional distilled $\mathrm{H}_{2} \mathrm{O}$.

6. Place resuspended meat in a clean, plastic screw-capped container. Add glass beads (marble sized). Replace cap and shake vigorously $(\sim 1 \mathrm{~min})$. (Note that we also tried this without beads, and it works almost as well.)

7. Filter homogenate (the mechanically disrupted tissue in solution) through cheesecloth three times. Collect and save the filtrate. Be sure that there are two or more layers of cheesecloth that are placed over the top of each beaker and that these layers are firmly secured with rubber bands. Also, ensure that there is a dimple in each cheesecloth cover (refer to Figure $2 c^{*}$ ).

8. Cover the resultant homogenate with plastic wrap and place at $4^{\circ} \mathrm{C}$ (in a refrigerator not used for food storage) overnight. This allows the meat slurry to settle to the bottom and the supernatant to rise to the top (Figure $2 \mathrm{~d}$ ). A centrifuge is not needed if this step is taken.

Day $2(\sim 20 \mathrm{~min})$.

Flotation Protocol (Figure 2, $d-f$ ). For the flotation protocol, conduct the steps listed below:

1. Make a supersaturated sucrose solution (i.e., add $60 \mathrm{~g}$ of sucrose to $20 \mathrm{ml}$ of PBS and adjust the volume of PBS until the solution is at $100 \mathrm{ml})$. Stir well to get the sucrose into solution. This produces a $60 \%$ sucrose solution.

2. Place the supernatant (Figure $2 \mathrm{~d}$ ) and saturated sugar solution in a 1:1 ratio in a clean, plastic screw-capped tube or container. Remember that the supernatant has floated to the top of the meat slurry (Figure 2d).

3. Shake vigorously.

4. Allow the solution to settle at $4^{\circ} \mathrm{C}$ overnight. The cysts and eggs will float to the top of the sugar solution (Figure 2f, beaker at left).

Day 3 ( $\sim 40 \mathrm{~min})$

Slide Preparation (Figure 2, $g-i$ ). For slide preparation, conduct the steps listed below:

1. Clean slides (70\% ethanol and deionized water); dry and cover until the slides are used.

2. Take a drop of the cysts and eggs that have floated to the top of the beaker and place them on a clean, dry slide (Figure $2 \mathrm{~g}$ ). The cysts and eggs will have floated to the top of the sugar solution. Place the drop on a slide (Figure $2 \mathrm{~g}$ ), smear the drop by using a second slide (Figure $2 \mathrm{~h}$ ), and air-dry the slide. The amount of time needed to dry the sample will vary based on drop size. The eggs have a sandy or gritty appearance and they are off-white or gray. To demonstrate their size and color, we floated a coverslip directly on top of one of the sucrose flotation samples and took a picture of the recovered cysts and eggs (Figure 2f, inset).

3. Place the slide in a beaker that has been prefilled with methanol (Figure 2i). Leave the slide in the methanol-filled beaker for 5 min at room temperature. This will fix and permeabilize the sample (i.e., make the dye more readily stain the cell). Methanol should be disposed of in an approved container.

4. Remove the slide with tweezers, being careful not to touch the liquid.

5. Place the fixed slide directly in a beaker filled with Giemsa solution (1:20 dilution), and leave slide in the stain for $30 \mathrm{~min}$. We suggest that during the incubation, one or more of the topics suggested in the Supplemental Material can be discussed. Make up a fresh Giemsa dilution from concentrate immediately before use.

6. Wash the slide two times by gently submerging the slide into a beaker filled with distilled water (Figure 2i). For more information on Giemsa, see Supplemental Material. 
7. Air-dry the slide for $15 \mathrm{~min}$. Coverslip and mounting media are optional but highly recommended.

Day 4 ( $\sim 50 \mathrm{~min})$.

Sample Viewing using Light Microscopy (Figure 3).

View sample at low magnification $(10 \times, 20 \times$, or $40 \times$ is fine). No immersion oil is needed.

Relevant Website Information. Useful images and information are available from the following websites; some sites show graphic depictions of parasitology symptoms: www.dpd.cdc.gov/dpdx/ default.htm, www.eden.rutgers.edu/ tavisa/Notes/Eggs.jpg, www.atlas.or.kr/index.html, and http://pathmicro.med.sc.edu/ book/parasit-sta.htm.

For government recommendations on biosafety, see Chosewood and Wilson, 2007). The full laboratory biosafety document (pdf format) can be downloaded at www.cdc.gov/od/ohs/biosfty/ bmbl5/bmbl5toc.htm.

\section{RESULTS AND DISCUSSION}

\section{Developing an Accessible Assay}

To develop a suitable assay, several factors were taken into consideration during meat selection. First, we chose only meats commonly cooked within home kitchens. This makes the assay more accessible because these meats are readily available at local grocery stores. Furthermore, it has the benefit of making the assay a more effective teaching tool because these types of ordinary foods are likely to be encountered by students from all walks of life. For example, $T$. gondii cysts are enriched in brain tissue; however, it is unlikely that brain is on the menu in a typical household. Therefore, it was eliminated as a source of cysts. Second, we selected meat from animals that are infected in high numbers with T. gondii (i.e., lamb and pig) to increase the chance of successfully isolating tissue cysts. Third, to increase the flexibility and hence accessibility of this assay for a wide student audience, we considered the safety factor when selecting meats. T. gondii is a BSL-2 organism. The CDC has established four biosafety levels: BSL-1, no known or minimal potential hazard of exposure to infectious agents; BSL-2, moderate potential hazard, low risk of exposure to infectious agents; BSL-3, moderate risk of exposure to agents that can cause serious or potentially lethal disease; and BSL-4, high individual risk of exposure to dangerous or exotic agents that cause life-threatening disease (Chosewood and Wilson, 2007). The National Science Education Leadership Association (www.nsela.org/publications/safescience/ss- article18.html) states that secondary schools should use BSL-1 experiments exclusively. Therefore, even though T. gondii is found in meat brought into homes every day and it does not usually cause disease in healthy individuals, we developed an alternative meat protocol. When considering this optional protocol, we selected an animal known to be largely refractory to cyst formation by $T$. gondii, a common feed animal, and yet enriched in high numbers of related Apicomplexa capable of forming readily recognizable tissue cysts. Cattle were selected as the alternative meat source. T. gondii does not form cysts in detectable amounts in cattle, and cattle are highly infected with the apicomplexan parasites N. caninum (Dubey et al., 2007) and S. cruzi (More et al., 2007).

After meat selection, we faced the challenge of efficiently, quickly, and inexpensively disrupting the meat to release the cysts. We chose to use a combination of detergent and mechanical disruption (Figure 2b). Again, we took into account the biosafety aspect. Although using a blender is the best and simplest method, use of a blender could potentially create aerosols. Therefore, we also created an alternative approach that uses screw-capped tubes containing glass beads. This breaks up the tissue without the creation of breathable aerosols. Some methodologies for T. gondii isolation suggest digesting the meat with the enzyme pepsin as opposed to using the detergent Tween 80 (Dubey, 1992, 1998; Garcia et al., 2006). After careful comparison, however, we concluded that Tween 80 was more practical for our needs. The Tween 80 was cheaper, did not require multiple heat cycles, and was less time-consuming to incorporate than the enzyme protocol.

Once the tissue was disrupted, the cysts needed to be separated from large cellular debris. We processed the meat homogenate by filtration through cheesecloth. The cheesecloth covered the top of a beaker, and it was secured by a rubber band. However, the cheesecloth should not be stretched tightly across the top of the beaker (Figure 2c, arrows). It is important to create a significant dimple in the middle of the cheesecloth covering the beaker (Figure $2 \mathrm{c}^{*}$ ). If this is not done, the meat homogenate quickly spills over the sides of the beaker, moving through the cheesecloth by capillary action (Figure 2c, far left beaker). We found the filtration step to be most efficacious given the viscous nature of the meat homogenates. This is particularly true for meats such as pork sausage and ground beef that are high in fat a.

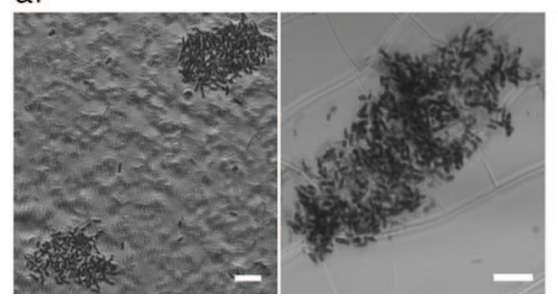

b.

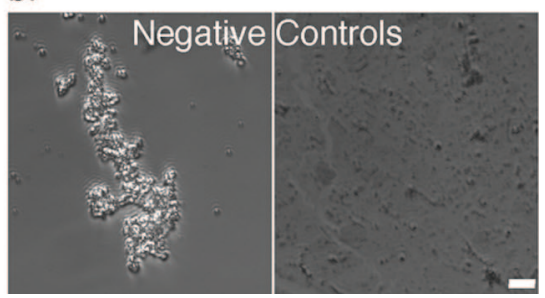

Figure 3. Isolated apicomplexan cysts. (a) Apicomplexan cysts (broken or ruptured) are releasing their bradyzoites. Samples were fixed and processed as described in Materials and Methods. Lamb contained the most cysts. Cysts were also observed in pork. Beef contained N. caninum and S. cruzi tissue cysts. Left, an example of an apicomplexan cyst obtained from the sucrose flotation protocol. Right, an example of a broken cyst isolated using the Percoll protocol. (b) Negative controls. Blank unwashed slides were processed for imaging. The left negative control slide shows a typical piece of debris. The right negative control slide shows what the stain looks like when it has dried on glass. Bars, $10 \mu \mathrm{m}$. 
content. This stage of the experiment also represents a good stopping place for laboratories with short periods. The filtrate may be covered and allowed to stand overnight at $4^{\circ} \mathrm{C}$ (refrigerate). This allows the slurry to settle and the supernatant to rise to the top (Figure 2d), eliminating the need for a centrifuge spin. Both centrifugation and the overnight incubation accomplish the same task, separation of components based on their size and density. Centrifugation is simply faster.

After the initial separation, we considered methods for obtaining T. gondii cysts. We arrived at two methodologies: one method to purify and the other method to enrich for $T$. gondii cysts. The first method, Percoll density gradient analysis, separates components solely on the basis of their buoyant density, resulting in a finer separation of materials. $T$. gondii cysts have a known buoyant density of $1.056 \mathrm{~g} / \mathrm{cm}^{3}$ (Figure 2e, white arrow). The cysts obtained from this preparation were more concentrated in number and freer of contaminants than the cysts obtained in the sucrose flotation protocol (Figure 3a, right). In the second method, we enriched rather than purified for $T$. gondii cysts. What that meant in practical terms is that the sample contained many types of apicomplexan cysts (Figure 3a, left) and also other contaminants. The cysts themselves look like a fine grain or grit. To obtain a picture of the sample, we floated a coverslip directly on top of one of the sucrose flotation samples, and then we took a picture of the recovered cysts and eggs (Figure 2f, inset).

\section{Imaging Apicomplexan Cysts}

Bradyzoites (brady=slow in Greek), the slow-growing form of T. gondii, are found within tissue cysts (Figure 1). Tissue cysts are long-lasting; they can persist for the lifetime of the host. Tissue cysts vary in size; they can range from 5 to 100 $\mu \mathrm{m}$ (in intramuscular tissue; Dubey et al., 1998). The tissue cyst wall is thin $(0.5 \mu \mathrm{m})$. Our isolation protocol typically disrupts the cyst wall; consequently, trypsin is often unnecessary (Figure 3a). Compared with tachyzoites (tachy=fast), the fast-growing form of the parasite, bradyzoites are more slender, and their nucleus is slightly more posterior (as revealed by Giemsa staining). The apicomplexan cysts that we isolated from lamb and pork possessed the expected morphology of bradyzoite tissue cysts (Figure 3a).

In terms of imaging, it is important to clean the microscope slides with $70 \%$ ethanol and water before fixation. This rather trivial step is important because to inexperienced students, dirt and airborne contaminants (Figure 3b, right, negative controls) might be difficult to discriminate from certain parasite eggs or cysts. We emphasize that although it is possible to simply take a drop of the cyst sample and place it on a slide and allow it to dry, the smear method depicted in Figure $2 \mathrm{~h}$ is a more effective method for imaging samples. The smear spreads out the isolated organisms and eggs or cysts. This is particularly important to do for the sucrose flotation method, because the number of organisms obtained is very high.

\section{SUMMARY}

Our goal was to design a bench experiment tailored to the environment of advanced high school biology, college fresh- man biology, and nonmajor labs. To accomplish this goal, we developed a scientific protocol for isolation of apicomplexan cysts from store-bought meat by adapting existing protocols (Cornelissen et al., 1981; Blewett et al., 1983; Dubey, 1992, 1998; Omata et al., 1997; Garcia et al., 2006). The majority of buffers used were common chemicals found in many introductory biology laboratories, and the protocol is simple enough for a freshman to use.

What we found to be most challenging was modifying the experiment to fit time constraints imposed by a typical high school schedule. This experiment takes $\sim 3 \mathrm{~h}$. We found that the lab could be split up into $4 \mathrm{~d}$ for high school and $2 \mathrm{~d}$ for college. The teacher should make some initial preparations (i.e., making buffers and solutions) to shorten the lab period. We suggest that students split up into at least five groups of six students each. The students should be encouraged to compare and share their results. We recommend that the students spend time researching $T$. gondii statistics on the CDC website (www.cdc.gov) and reading newspapers to identify related topics, such as food recalls as a result of tainted product. Specifically, we suggest creating a quiz to test their knowledge of food safety and administering it before and after the exercise. We would also suggest including a follow-up discussion section investigating student response to this assignment. For example, how has completing this laboratory exercise affected their opinions about food, science, and laboratory experiments? We have listed several very useful websites in Materials and Methods. These sites are extremely helpful in providing pictures for identification purposes and background information on the parasites themselves.

In addition to T. gondii, numerous other organisms (i.e., multiple types of nematodes and tapeworms) can be observed by sampling the bottom of the meat slurry instead of the supernatant. Although we had multiple years of experience at the lab bench, we found that we were surprised by the abundance and diversity of organisms in meat. It provoked us to ask the question: "Just who is eating who?" Although many of these pathogens are species specific and thus not a health threat to human (i.e., N. caninum), some of the organisms, such as T. gondii, are human pathogens. We realized that it reinvigorated our interest in the topic, and we were fascinated by the images in the microscope. Some of us could not eat meat for a week after doing the experiment. This realization validated for us the idea that science at the bench as opposed to simple book learning is essential for biology and nonmajors students alike. It drives home the point that well-prepared foods (washing and cooking) are a cornerstone of food safety. Carrying out this experiment familiarizes students with the microscope and more advanced isolation techniques such as the use of Percoll. It introduces some concepts about parasitology and food safety. It can be incorporated into a lesson plan covering infectious agents or health. It meets Indiana secondary school standards for advanced life sciences (standard 2: health, safety, and microbiology of food), and human anatomy and physiology (standard 10: immune mechanisms). Completion of this exercise also meets national educational standards for secondary school in the topics science as inquiry, interdependence of organisms, personal and community health, and science as a human endeavor (National 
Committee on Science Education Standards and Assessment, 1996).

Upon completion of this exercise, the following positive outcomes are expected for students: 1) gain confidence about ability to carry out labwork and stimulate curiosity about pursuing other science classes, 2) obtain insight about health and safety of food and provide students the chance to critically evaluate articles in the newspaper regarding food recalls, and 3) change student behavior in terms of food choice, food preparation behavior, or both.

In sum, the ultimate outcome of this exercise is for students to apply the procedure to investigate a real and meaningful problem and, as a result, to describe organisms found in the meat supply, the hazards they pose, and procedures for avoiding the hazard. Because there is a compelling repulsion factor, there is a strong possibility that students would also change their behavior (either food choices or food preparation behavior). For teachers, it represents an innovative teaching strategy that provides a relatively inexpensive laboratory experience with direct student involvement that meets several national education standards.

\section{ACKNOWLEDGMENTS}

We thank Kevin Barry (Kaneb Center for Teaching and Learning) for support and advice. We extend a special thanks to Larry Mordan (a very dedicated high school biology teacher), Jennifer Robichaud (a great veterinarian parasitologist), and Karen Kalumuck (Exploratorium Teacher Institute) for invaluable insight and suggestions regarding this work. E.F. was supported through the Research Experiences for Teachers at Notre Dame program, a program funded by the National Science Foundation. K.M.H. was supported though funds from the Ellison Medical Foundation New Scholar in Infectious Disease program and a University of Notre Dame Faculty Research Program Award.

\section{REFERENCES}

Abrahamsen, M. S. et al. (2004). Complete genome sequence of the apicomplexan, Cryptosporidium parvum. Science 304, 441-445.

Black, M. W., and Boothroyd, J. C. (2000). Lytic cycle of Toxoplasma gondii. Microbiol. Mol. Biol. Rev. 64, 607-623.

Blewett, D. A., Miller, J. K., and Harding, J. (1983). Simple technique for the direct isolation of toxoplasma tissue cysts from fetal ovine brain. Vet Rec 112, 98-100.

Chosewood, L. C., and Wilson, D. E. (2007). Biosafety in Microbiological and Biomedical Laboratories, 5th ed. U.S. Department of Health and Human Services. Centers for Disease Control and Prevention, and National Institutes of Health, Washington, DC: U.S. Government Printing Office.

Cornelissen, A. W., Overdulve, J. P., and Hoenderboom, J. M. (1981). Separation of Isospora (Toxoplasma) gondii cysts and cystozoites from mouse brain tissue by continuous density-gradient centrifugation. Parasitol. 83, 103-108.

Cox, F. E. (2002). History of human parasitology. Clin. Microbiol. Rev. 15, 595-612.

Dobell, C. E. (1960). Antony van Leeuwenhoek and his "Little Animals," New York: Dover Publications.

Dubey, J. P. (1992). Isolation of Toxoplasma gondii from a naturally infected beef cow. J. Parasitol. 78, 151-153.
Dubey, J. P. (1998). Refinement of pepsin digestion method for isolation of Toxoplasma gondii from infected tissues. Vet. Parasitol. 74, 75-77.

Dubey, J. P., Lindsay, D. S., and Speer, C. A. (1998). Structures of Toxoplasma gondii tachyzoites, bradyzoites, and sporozoites and biology and development of tissue cysts. Clin. Microbiol. Rev. 11, 267-299.

Dubey, J. P., Schares, G., and Ortega-Mora, L. M. (2007). Epidemiology and control of neosporosis and Neospora caninum. Clin. Microbiol. Rev. 20, 323-367.

Fayer, R., Dubey, J. P., and Lindsay, D. S. (2004). Zoonotic protozoa: from land to sea. Trends Parasitol. 20, 531-536.

Ferguson, M. A., Homans, S. W., Dwek, R. A., and Rademacher, T. W. (1988). Glycosyl-phosphatidylinositol moiety that anchors Trypanosoma brucei variant surface glycoprotein to the membrane. Science 239, 753-759.

Garcia, J. L., Gennari, S. M., Machado, R. Z., and Navarro, I. T. (2006). Toxoplasma gondii: detection by mouse bioassay, histopathology, and polymerase chain reaction in tissues from experimentally infected pigs. Exp. Parasitol. 113, 267-271.

Gardner, I. A. (2004). An epidemiologic critique of current microbial risk assessment practices: the importance of prevalence and test accuracy data. J. Food Prot. 67, 2000-2007.

Graham, D. A., Calvert, V., Whyte, M., and Marks, J. (1999). Absence of serological evidence for human Neospora caninum infection. Vet. Rec. 144, 672-673.

Hill, D. E., Chirukandoth, S., and Dubey, J. P. (2005). Biology and epidemiology of Toxoplasma gondii in man and animals. Anim. Health Res. Rev. 6, 41-61.

Innes, E. A., Bartley, P. M., Maley, S. W., Wright, S. E., and Buxton, D. (2007). Comparative host-parasite relationships in ovine toxoplasmosis and bovine neosporosis and strategies for vaccination. Vaccine 25, 5495-5503.

Köhler, S., Delwiche, C. F., Denny, P. W., Tilney, L. G., Webster, P., Wilson, R. J., Palmer, J. D., and Roos, D. S. (1997). A plastid of probable green algal origin in apicomplexan parasites. Science 275, $1485-1489$.

Lang-Unnasch, N., Reith, M. E., Munholland, J., and Barta, J. R. (1998). Plastids are widespread and ancient in parasites of the phylum Apicomplexa. Int. J. Parasitol. 28, 1743-1754.

Levine, N. D. (1977). Taxonomy of Toxoplasma. J. Protozool. 24, $36-41$.

Levine, N. D. (1988). Progress in taxonomy of the apicomplexan protozoa. J. Protozool. 35, 518-520.

Marquardt, W. C., Demaree, R. S. and Grieve, R. B. (2000). Parasitology and Vector Biology, London, United Kingdom: Harcourt/ Academic Press.

McFadden, G. I., Reith, M. E., Munholland, J., and Lang-Unnasch, N. (1996). Plastid in human parasites. Nature 381, 482.

McFadden, G. I., and Roos, D. S. (1999). Apicomplexan plastids as drug targets. Trends Microbiol. 7, 328-333.

More, G., Basso, W., Bacigalupe, D., Venturini, M. C., and Venturini, L. (2007). Diagnosis of Sarcocystis cruzi, Neospora caninum, and Toxoplasma gondii infections in cattle. Parasitol. Res. 102, 671-675.

National Committee on Science Education Standards and Assessment (1996). National Science Education Standards. National Committee on Science Education Standards and Assessment, Washington, DC: National Academies Press.

Omata, Y., Taka, A., Terada, K., Koyama, T., Kanda, M., Saito, A., and Dubey, J. P. (1997). Isolation of coccidian enteroepithelial stages of Toxoplasma gondii from the intestinal mucosa of cats by Percoll density-gradient centrifugation. Parasitol. Res. 83, 574-577. 
Pfluger, S. L., Goodson, H. V., Moran, J. M., Ruggiero, C. J., Ye, X., Emmons, K. M., and Hager, K. M. (2005). Receptor for retrograde transport in the apicomplexan parasite Toxoplasma gondii. Eukaryot. Cell 4, 432-442.

Roberts, L. S., and Schmidt, G. D. (2000). Phylum Apicomplexa: gregarines, coccicidia, and related organisms. In: Gerald D. Schmidt \& Larry S. Roberts' Foundations of Parasitology, 6th ed., ed. L. S. Roberts and J. Janovy, Jr., New York: McGraw-Hill Companies.

Thurston-Enriquez, J. A., Gilley, J. E., and Eghball, B. (2005). Microbial quality of runoff following land application of cattle manure and swine slurry. J. Water Health 3, 157-171.
Thurston-Enriquez, J. A., Watt, P., Dowd, S. E., Enriquez, R., Pepper, I. L., and Gerba, C. P. (2002). Detection of protozoan parasites and microsporidia in irrigation waters used for crop production. J. Food Prot. 65, 378-382.

Waller, R. F., and McFadden, G. I. (2005). The apicoplast: a review of the derived plastid of apicomplexan parasites. Curr. Issues Mol. Biol. 7, 57-79.

Zhu, G., Marchewka, M. J., and Keithly, J. S. (2000). Cryptosporidium parvum appears to lack a plastid genome. Microbiology 146, 315321. 